\title{
SOLUTION OF AN INTEGRAL EQUATION
}

\section{by J. S. LOWNDES}

(Received 4 August, 1976) equation

1. The integral equation. In this paper we obtain the general solution of the

$$
\int_{0}^{a} f(x) k_{z}(x, y) d x=g(y) \quad(0<y<a)
$$

whose kernel is

$$
k_{z}(x, y)=\frac{\sigma \delta x^{\sigma \eta-1} y^{\delta \mu}}{\Gamma(1-\alpha) \Gamma(1-\beta)} \int_{z}^{\infty} \frac{t^{\sigma(\alpha-\eta)+\delta(\beta-\mu)-1}}{\left(t^{\sigma}-x^{\sigma}\right)^{\alpha}\left(t^{\delta}-y^{\delta}\right)^{\beta}} \phi(t) d t
$$

where $0<\alpha, \beta<1, \mu, \eta, \sigma>0, \delta>0$, are real parameters, $z=\max (x, y), \phi(t)$ and $g(y)$ are prescribed functions and $f(x)$ is to be determined.

Using simple generalisations of the Erdélyi-Kober operators of fractional integration it is shown, in a purely formal manner, that the solution of the equation can be found in terms of the solution of a Fredholm integral equation of the second kind. In particular, when the upper limit $a$ is taken to be infinity, an exact solution of the equation is found which is in agreement with that obtained by Sneddon [5, p. 49] when $\sigma=\delta=2$.

As an example of the method a generalisation of an integral equation, which occurs in the solution of mixed boundary value problems involving the wave equation, is solved.

2. The integral operators. We shall use the slightly extended forms of the ErdélyiKober operators defined in [1] by

$$
\begin{array}{rlr}
I_{\eta, \alpha}(a, x: \sigma) f(x) & =\frac{\sigma x^{-\sigma(\alpha+\eta)}}{\Gamma(\alpha)} \int_{a}^{x}\left(x^{\sigma}-t^{\sigma}\right)^{\alpha-1} t^{\sigma(\eta+1)-1} f(t) d t & (\alpha>0) \\
& =\frac{x^{1-\sigma(\alpha+\eta+1)}}{\Gamma(1+\alpha)} \frac{d}{d x} \int_{a}^{x}\left(x^{\sigma}-t^{\sigma}\right)^{\alpha} t^{\sigma(\eta+1)-1} f(t) d t & (-1<\alpha<0) \\
K_{\eta, \alpha}(x, b: \sigma) f(x) & =\frac{\sigma x^{\sigma \eta}}{\Gamma(\alpha)} \int_{x}^{b}\left(t^{\sigma}-x^{\sigma}\right)^{\alpha-1} t^{\sigma(1-\alpha-\eta)-1} f(t) d t \quad(\alpha>0), \\
& =-\frac{x^{\sigma(\eta-1)+1}}{\Gamma(1+\alpha)} \frac{d}{d x} \int_{x}^{b}\left(t^{\sigma}-x^{\sigma}\right)^{\alpha} t^{\sigma(1-\alpha-\eta)-1} f(t) d t & (-1<\alpha<0)
\end{array}
$$

where $a<x<b$ and $\sigma>0$.

Glasgow Math. J. 19 (1978) 69-73. 
The inverse operators are

$$
\begin{aligned}
I_{\eta, \alpha}^{-1}(a, x: \sigma) f(x) & =I_{\eta+\alpha,-\alpha}(a, x: \sigma) f(x), \\
K_{\eta, a}^{-1}(x, b: \sigma) f(x) & =K_{\eta+\alpha,-\alpha}(x, b: \sigma) f(x) .
\end{aligned}
$$

Use will also be made of the operators $L_{\eta, \alpha}(0, x: \sigma)$ and $M_{\eta, \alpha}(x, b: \sigma)$ which when $|\alpha|<1$ are defined in $[\mathbf{1}]$ to be

$$
\begin{aligned}
L_{\eta, \alpha}(0, x: \sigma) f(x) & =-I_{\eta+\alpha,-\alpha}(0, a: \sigma) I_{\eta, \alpha}(0, x: \sigma) f(x) \quad(0<a<x), \\
= & \frac{\sigma \sin (\alpha \pi)}{\pi} \frac{x^{-\sigma \eta}}{\left(x^{\sigma}-a^{\sigma}\right)^{\alpha}} \int_{0}^{a} \frac{u^{\sigma(\eta+1)-1}\left(a^{\sigma}-u^{\sigma}\right)^{\alpha}}{x^{\sigma}-u^{\sigma}} f(u) d u, \\
M_{\eta, \alpha}(x, b: \sigma) f(x) & =K_{\eta, \alpha}^{-1}(x, a: \sigma) K_{\eta, \alpha}(a, b: \sigma) f(x) \quad(x<a<b), \\
& =\frac{\sigma \sin (\alpha \pi)}{\pi} \frac{x^{\sigma(\alpha+\eta)}}{\left(a^{\sigma}-x^{\sigma}\right)^{\alpha}} \int_{a}^{b} \frac{u^{\sigma(1-\alpha-\eta)-1}\left(u^{\sigma}-a^{\sigma}\right)^{\alpha}}{u^{\sigma}-x^{\sigma}} f(u) d u .
\end{aligned}
$$

3. Solution of the integral equation. Substituting for the kernel from equation (2) into equation (1) we find that the integral equation becomes

$$
\int_{0}^{y} f(x) k_{y}(x, y) d x+\int_{y}^{a} f(x) k_{x}(x, y) d x=g(y) \quad(0<y<a) .
$$

Interchanging the order of the integrations and using the definitions (3) and (5) the above equation can be written in the operational form

$$
K_{\mu, 1-\beta}(y, a: \delta) F(y)+K_{\mu, 1-\beta}(a, \infty: \delta) F_{1}(y)=g(y) \quad(0<y<a),
$$

where

$$
\begin{gathered}
F(x)=\phi(x) I_{\eta-1,1-\alpha}(0, x: \sigma) f(x), \\
F_{1}(x)=\phi(x) I_{\eta-1,1-\alpha}(0, a: \sigma) f(x) .
\end{gathered}
$$

On using the inversion formula (8) we find that equation (12) becomes

$$
F(x)+M_{\mu, 1-\beta}(x, \infty: \delta) F_{1}(x)=K_{\mu, 1-\beta}^{-1}(x, a: \delta) g(x) \quad(0<x<a),
$$

where the operator $M_{\mu, 1-\beta}(x, \infty: \delta)$ is defined by equation (10).

Applying the inversion formula (7) to equation (13) we get

$$
f(x)=I_{\eta-\alpha, \alpha-1}(0, x: \sigma)\left[\frac{F(x)}{\phi(x)}\right],
$$

which together with equation (14) shows that the function $F_{1}(x)$ is given in terms of $F(x)$ by

$$
F_{1}(x)=-\phi(x) L_{\eta-\alpha, \alpha-1}(0, x: \sigma)\left[\frac{F(x)}{\phi(x)}\right] .
$$

where $L_{\eta-\alpha, \alpha-1}(0, x: \sigma)$ is defined by equation (9). 
Eliminating $F_{1}(x)$ between equations (15) and (17) we find that $F(x)$ satisfies the Fredholm integral equation of the second kind

$$
F(x)-M_{\mu, 1-\beta}(x, \infty: \delta)\left\{\phi(x) L_{n-\alpha, \alpha-1}(0, x: \sigma)\left[\frac{F(x)}{\phi(x)}\right]\right\}=K_{\mu, 1-\beta}^{-1}(x, a: \delta) g(x),
$$

where $0<x<a$.

When $F(x)$ has been determined from this equation the solution function $f(x)$ of the integral equation (1) can be found from equation (16).

Letting $a$ tend to infinity and using the result $\operatorname{Lim}_{a \rightarrow \infty} M_{\eta, \alpha}(x, \infty: \delta) G(x)=0$, we find from equations (16) and (18) that the solution of the equation

$$
\int_{0}^{\infty} f(x) k_{z}(x, y) d x=g(y) \quad(0<y<\infty),
$$

is

$$
f(x)=I_{\eta-\alpha, \alpha-1}(0, x: \sigma)\left[\frac{1}{\phi(x)} K_{\mu-\beta+1, \beta-1}(x, \infty: \delta) g(x)\right] .
$$

If $0<\alpha, \beta<1$, this solution, when written out in detail, can easily be shown to be

$$
f(x)=-\frac{x^{1-\sigma \eta}}{\Gamma(\alpha) \Gamma(\beta)} \frac{d}{d x} \int_{0}^{x} \frac{t^{\sigma(\eta-\alpha+1)+\delta(\mu-\beta)}}{\phi(t)\left(x^{\sigma}-t^{\sigma}\right)^{1-\alpha}} d t \frac{d}{d t} \int_{t}^{\infty} \frac{y^{\delta(1-\mu)-1}}{\left(y^{\delta}-t^{\delta}\right)^{1-\beta}} g(y) d y,
$$

which is equivalent to the solution obtained by Sneddon [5] when $\sigma=\delta=2$.

4. An example. Consider the Wiener-Hopf type of integral equation

$$
\int_{0}^{a} K_{\lambda}(p|x-y|) \frac{u(x)}{(|x-y|)^{\lambda}} d x=v(y) \quad(0<y<a),
$$

where $\operatorname{Re}(p)>0,|\lambda|<\frac{1}{2}, v(y)$ is a known function and $u(x)$ is to be found. $K_{\lambda}(w)$ is the modified Bessel function of the second kind [2, p. 85]

$$
\Gamma\left(\frac{1}{2}+\lambda\right) K_{\lambda}(w)=\pi^{1 / 2}\left(\frac{w}{2}\right)^{\lambda} \int_{0}^{\infty} e^{-w \cosh t}(\sinh t)^{2 \lambda} d t,
$$

where $\operatorname{Re}(w)>0$ and $2 \lambda>-1$.

When $\lambda=0$ the integral equation reduces to the well known equation

$$
\frac{\pi i}{2} \int_{0}^{a} H_{0}^{(1)}(q|x-y|) u(x) d x=v(y) \quad(\operatorname{Im}(q)>0,0<y<a),
$$

where $H_{0}^{(1)}(w)$ is the Bessel function of the third kind of order zero, which occurs in the solution of mixed boundary value problems involving the wave equation [3]. 
We shall make use of the following representation of the modified Bessel function

$$
(2 p)^{\lambda} \Gamma\left(\frac{1}{2}-\lambda\right) K_{\lambda}(p|x-y|)=\pi^{1 / 2}(|x-y|)^{\lambda} e^{p(x+y)} I_{z}(x, y),
$$

where

$$
I_{z}(x, y)=\int_{z}^{\infty} \frac{e^{-2 p t}}{[(t-x)(t-y)]^{\lambda+1 / 2}} d t
$$

$\operatorname{Re}(p)>0,2 \lambda<1$ and $z=\max (x, y)$.

The above expression is a generalisation of a result given by Noble [3, p. 230] and is easily verified by considering the integral (26) when $x>y$ and $y>x$ separately and showing that in each case equation (25) reduces to equation (23).

Making the substitutions

$$
u(x)=e^{-p x} f(x), \quad v(y)=e^{p y} g(y),
$$

we find that equation (22) takes on the same form as equation (1) with the kernel

$$
\begin{aligned}
k_{z}(x, y) & =\frac{e^{-p(x+y)}}{(|x-y|)^{\lambda}} K_{\lambda}(p|x-y|) \\
& =\frac{1}{\left[\Gamma\left(\frac{1}{2}-\lambda\right)\right]^{2}} \int_{z}^{\infty} \frac{t^{2 \lambda-1} \phi(t)}{[(t-x)(t-y)]^{\lambda+1 / 2}} d t,
\end{aligned}
$$

where $z=\max (x, y)$ and we have written

$$
\phi(t)=\pi^{1 / 2} \Gamma\left(\frac{1}{2}-\lambda\right)(2 p)^{-\lambda} t^{1-2 \lambda} e^{-2 p t} .
$$

This kernel is identical with that given by equation (2) when $\sigma=\delta=\eta=1, \mu=0$ and $\alpha=\beta=\lambda+\frac{1}{2}$.

Hence from the general results (16) and (18) and the definitions (27) and (29) we find that the solution of equation (22) is

$$
\pi^{1 / 2} \Gamma\left(\frac{1}{2}-\lambda\right) u(x)=(2 p)^{\lambda} e^{-p x} I_{1 / 2-\lambda, \lambda-1 / 2}(0, x: 1)\left[x^{2 \lambda-1} e^{2 p x} U(x)\right] \quad(0<x<a),
$$

where $U(x)$ is the solution of the Fredholm integral equation

$$
\begin{aligned}
U(x)-M_{0,1 / 2-\lambda}(x, \infty: 1)\left\{x^{1-2 \lambda} e^{-2 p x} L_{1 / 2-\lambda, \lambda-1 / 2}(0, x: 1)\right. & {\left.\left[x^{2 \lambda-1} e^{2 p x} U(x)\right]\right\} } \\
& =K_{0,1 / 2-\lambda}^{-1}(x, a: 1)\left[e^{-p x} v(x)\right] .
\end{aligned}
$$

When $|\lambda|<\frac{1}{2}$ it can easily be shown that the solution can be written in the form

$$
e^{p x} u(x)=\frac{\cos (\lambda \pi)}{\pi} \frac{d}{d x} \int_{0}^{x} \frac{U^{*}(t)}{(x-t)^{1 / 2-\lambda}} d t
$$

where $U^{*}(t)$ satisfies the integral equation

$$
U^{*}(t)+\frac{1}{\pi^{2}} \int_{0}^{a} U^{*}(s) T(s, t) d s=-\frac{(2 p)^{\lambda} e^{2 p t}}{\pi^{1 / 2} \Gamma\left(\frac{1}{2}+\lambda\right)} \frac{d}{d t} \int_{t}^{a} \frac{e^{-p y} v(y)}{(y-t)^{1 / 2-\lambda}} d y \quad(0<t<a)
$$


and $T(s, t)$ is the kernel

$$
T(s, t)=\frac{\cos ^{2}(\lambda \pi)}{[(a-s)(a-t)]^{1 / 2-\lambda}} \int_{a}^{\infty} \frac{(\xi-a)^{1-2 \lambda} e^{-2 p(\xi-t)}}{(\xi-s)(\xi-t)} d \xi
$$

Finally, letting $a$ tend to infinity, we see that the solution of the integral equation

$$
\int_{0}^{\infty} K_{\lambda}(p|x-y|) \frac{u(x)}{(|x-y|)^{\lambda}} d x=v(y) \quad\left(|\lambda|<\frac{1}{2}, 0<y<\infty\right)
$$

is given by

$$
\pi^{3 / 2} \Gamma\left(\frac{1}{2}+\lambda\right) e^{p x} u(x)=-(2 p)^{\lambda} \cos (\lambda \pi) \frac{d}{d x} \int_{0}^{x} \frac{e^{2 p t}}{(x-t)^{1 / 2-\lambda}} d t \frac{d}{d t} \int_{t}^{\infty} \frac{e^{-p y} v(y)}{(y-t)^{1 / 2-\lambda}} d y,
$$

which when $\lambda=0$ agrees with the solution obtained by Noble and Peters [4] using a different method.

\section{REFERENCES}

1. J. S. Lowndes, Some triple integral equations, Pacific J. Math. 38 (1971) 515-521.

2. W. Magnus, F. Oberhettinger and R. P. Soni, Formulas and theorems for the special functions of mathematical physics, 3rd ed. (Springer-Verlag, 1966)

3. B. Noble, Methods based on the Wiener-Hopf technique, (Pergamon Press, 1958)

4. B. Noble and A. S. Peters, A multiplying factor technique for the solution of Wiener-Hopf equations, Proc. Edinburgh Math. Soc. 12 (1961) 119-122.

5. I. N. Sneddon, The use in mathematical physics of Erdélyi-Kober operators and some of their generalisations, Lecture notes in mathematics No. 457 (Springer-Verlag, 1975) 37-79.

Department of Mathematics

UNIVERSTTY OF STRATHCLYDE

GLASGOW. 